\title{
Education and public outreach on gravitational-wave astronomy
}

\author{
M. Hendry • C. Bradaschia - H. Audley - S. Barke • D. G. Blair • \\ N. Christensen - K. Danzmann - A. Freise - O. Gerberding • B. Knispel • \\ M. Lieser • I. Mandel • T. Moore • A. Stuver • B. Whiting
}

Received: 26 January 2014 / Accepted: 19 June 2014 / Published online: 19 July 2014

(C) Springer Science+Business Media New York 2014

\begin{abstract}
In this paper we summarise the presentations given during the "Education and Public Outreach on Gravitational-Wave Astronomy" parallel session at the GR20/Amaldi conference, held in Warsaw, July 2013. The talks presented demonstrate the wide range of education and public outreach activities being undertaken in the field of gravitational-wave astronomy-across science festivals, science education centers, junior schools and high schools, colleges and universities, via both face-toface delivery and (increasingly) the internet and social media.
\end{abstract}

This article belongs to the Topical Collection: The First Century of General Relativity: GR20/Amaldi10.

M. Hendry ( $\square)$

SUPA, School of Physics and Astronomy, University of Glasgow, Glasgow G12 8QQ, UK

e-mail: martin.hendry@glasgow.ac.uk

C. Bradaschia

INFN-Pisa, Largo Bruno Pontecorvo, 56127 Pisa, Italy

e-mail: carlo.bradaschia@pi.infn.it

H. Audley · S. Barke · K. Danzmann · O. Gerberding · B. Knispel · M. Lieserl

Max-Planck Institute for Gravitational Physics, Albert Einstein Institute,

Callinstrasse 38, 30167 Hannover, Germany

e-mail: oliver.gerberding@aei.mpg.de

D. G. Blair

AIGRC, The University of Western Australia (M013), 35 Stirling Highway,

Crawley, WA 6009, Australia

e-mail: david.blair@uwa.edu.au

N. Christensen

Department of Physics and Astronomy, Carleton College, Northfield, MN 55057, USA

e-mail: nchriste@carleton.edu 
Keywords Gravitational waves - General relativity $\cdot$ Public engagement $\cdot$ Physics education

\section{Introduction}

The field of gravitational-wave astronomy is relatively young but in recent years it has enjoyed a prominent and rapidly growing public profile. This has been fuelled by the general fascination that exists among formal and informal learners with extreme cosmic events - such as black holes, supernovae, gamma ray bursts, even the Big Bang itself — and their role as natural 'laboratories' with which to test our understanding of the laws of physics, and in particular the theories of Albert Einstein. In addition, the high profile of gravitational-wave astronomy has also been driven by exciting technological developments-such as the design, construction and operation of the ground-based laser interferometers LIGO, Virgo and GEO600 - that are pushing the boundaries of science and engineering.

Within the global scientific community of this nascent field a large number of successful education and public outreach programs have been established. At the GR20/Amaldi conference, which took place in Warsaw in July 2013, a parallel session was organised to showcase some of those programs. The session, which was convened by Martin Hendry and Carlo Bradaschia, featured a total of seven short presentationswith the speakers covering a wide spectrum of topics and activities in both formal and informal education, in ground- and space-based gravitational-wave astronomy, and spanning audiences and age groups that ranged from junior school to university undergraduates. Also noteworthy was the rich variety of methods of engagement highlighted by the speakers: from 'traditional' face to face contact at science centers and science festivals to extensive use of the internet via e.g. websites, social media channels and memes. In addition to the six presentations summarised in this paper, the seventh talk given at the session was by Bruce Allen (AEI, Hannover) on the "Einstein@Home" project. This talk emphasised the direct role that members of the public can now take, as so-called "Citizen Scientists", in the analysis of gravitational-wave data-a method of engagement which has already achieved impressive results in this field and which has enormous potential for even greater impact in the future.

\footnotetext{
A. Freise - I. Mandel

School of Physics and Astronomy, University of Birmingham, Edgbaston, Birmingham B15 2TT UK

e-mail:mandeli@bham.ac.uk

T. Moore

Department of Physics and Astronomy, Pomona College, Claremont, CA 91711, USA

A. Stuver

LIGO Livingston, 19100 Ligo Rd, Livingston, LA 70754, USA

e-mail: amber.stuver@ligo.org

B. Whiting

Department of Physics, University of Florida, 2001 Museum Rd, Gainesville, FL 32611, USA

e-mail: bernard@phys.ufl.edu
} 


\section{Education and public outreach: skills and media (Presenter: A. Stuver)}

Central to the discovery of new knowledge is to disseminate these findings. The main means of this dissemination is usually through publications and presentations to the academic community, but the public also has much to gain from the application of this new knowledge. Because of this, it is important to engage the public in education and outreach efforts, which also serve to humanize science and scientists while inspiring the public. However, it can be difficult to find outlets that are appropriate for an individual scientist's skills and availability. This presentation outlined the author's advice on useful skills and media to effectively perform outreach from her experience at the LIGO Science Education Center (co-located with the LIGO Livingston Observatory) working professionally in both gravitational-wave research and outreach. (A summary of some LIGO outreach efforts is reported here [5]).

\subsection{Skills}

Make your science accessible in any way you can. Learn effective translations of jargon into common language. For this, it is often helpful to think of how you would describe your work to a loved one with minimal technical training.

To become effective at communicating science, you must work at it and you will find your unique voice in the process. Enthusiasm is contagious: if you allow your excitement to be evident in your presentation, your audience will be excited too. It is absolutely acceptable to admit that you don't know the answer to a question; acknowledge the validity of the question, state why you are unsure of the answer and that you will try to find out the answer to the question. Keep up with current news stories, as these are often excellent examples of science as a process (an excellent example of this is the claim of the detection of superluminal neutrinos from the OPERA experiment).

Respect your audience, including religious beliefs, and treat your audience as an equal. Remember that your science may be perceived as being in conflict with others' religious beliefs (e.g. the Big Bang is in conflict with some creationist world views). Try to understand what part of your science causes the conflict and why. Make the distinction between questions of science ("How?") and questions of faith ("Why?"). Always be respectful and remember that you are the embodiment of science to many that you engage and you don't want to send them away feeling alienated.

\subsection{Media}

\subsubsection{Blogging}

Blogging allows you to write about your science while engaging a potentially large audience (your audience will grow over time as you continue to write, network with other bloggers, and promote your blog through social media). Through this medium, you can humanize scientists by writing about your life, inspire your readers by reporting on news in your research field (or related fields), and educate your audience by answering questions they ask of you. The author's blog can be accessed here [1]. 
While it may seem that no one reads your blog in the early days and months, the media is paying attention. They may pick up on news that you break on your blog and come to you for interviews for their media outlet (both of which have happened to the author [2] and more recently [3]).

\subsubsection{Teacher professional development}

Most teachers are required to do some form of continuing education and you can provide this service. If you don't feel that you have content appropriate for delivery to educators outside of higher education, themed 'Year of' events are excellent sources of this content as are the outreach offices of professional societies (the author has had positive experience with the American Physical Society, American Association of Physics Teachers, and the American Optical Society). Supportive funding is also often available through professional societies [4]. As you are determining content to deliver for your teacher professional development event, build relationships with local school and be sure to advertise your event early to attract the audience you want.

\subsubsection{Visit schools}

Most schools aren't sure of how to attract scientists to talk with their students and, in the author's experience, rarely turn away the offer of a visit. Before you visit, consider what content you want to present and its age appropriateness. Bring portable demonstrations, activities, computer simulations, videos, or anything that will engage the students. Most importantly, bring your enthusiasm about what you do. Think about discussing with the students how you became a scientist and why you study your specific subject.

If you can't visit a school in person, consider being a virtual presence through Skype, etc. This will still allow real-time interaction between students, you, and your science.

\subsubsection{Other ideas}

- Give talks to interested community groups.

- Have an open house at your institution.

- Develop classroom activities to connect concepts they are learning in class to current research.

- Work with the education department at your local university on ways to interact with pre-service teachers.

- Work with professional societies to develop educational materials for wide distribution.

\section{Teaching Einsteinian physics at school (Presenter: D. Blair)}

Between 1900 and 1920 discoveries by Einstein, Planck and others revolutionised physics. Evidence of the existence of photons led to quantum mechanics and evidence 
that space is curved confirmed the core prediction of Einsteins general theory of relativity. These discoveries, which together we define as Einsteinian physics led to a completely new understanding of space, time, gravity, matter and radiation. Today Einsteinian physics has been tested to high precision, and is the fundamental basis for gravitational wave astronomy. Einsteinian physics lies at the heart of modern technology such as mobile phones, and is essential for understanding modern astronomy and timekeeping. Despite these extraordinary developments, all based on science first conceptualised almost 100 years ago, physics in schools is still taught from a 19th Century Newtonian standpoint.

The unfamiliar mathematical basis of general relativity, combined with conceptual difficulties of interpreting it prior to about 1960 led to a general belief that Einsteinian physics was beyond the reach of ordinary people. Thus it has been avoided in school. We argue that we owe it to our children to teach them the modern paradigm of Einsteinian physical reality that today represents our best understanding of the universe. Furthermore, we argue that Einsteinian physics should be taught as a first language and not as a second language learnt in adulthood—it is necessary to teach the modern paradigm while children are forming the world view with which they conceptualise reality.

We present evidence that the modern paradigm can be presented accurately and quantitatively without resort to tensor calculus. Our preliminary evidence shows that the concepts of curved space, time dilation, four dimensional space-time and quantum weirdness are seen as neither confronting nor revolutionary when taught to students young enough to have not already formed the Newtonian world view of absolute space and Euclidean geometry.

In a series of pilot studies we have been investigating the ability of students aged 11-16 to comprehend Einsteinian physics. [6]. We have developed curriculum materials and teaching aids that bring Einsteinian physics vividly to life. We make extensive use of activity based learning and analogies. For example we use foam bullets as analog photons and use these to teach about photography, quantum uncertainty and the photoelectric effect. We use laser pointers for beautiful simple interference experiments which are interpreted in the context of photons. We teach experimental geometry on curved spaces to show how Euclidean geometry is a special case of a more general geometry. We use lycra sheets to illustrate the concept that "matter tells space how to curve and space tells matter how to move. We use this to investigate orbits, gravitational lensing and Newtons law of gravitation in the Einsteinian context. More advanced students undertake calculations of gravitational light deflection, time dilation, mass-energy, radiation pressure. A key part of the program at senior levels involves making the connection between Einsteinian physics and the Newtonian approximation, which lead into classical Newtonian physics curriculum content.

A most important part of our program is the development of professional development training programmes for school teachers, and testing whether teachers can attain a sufficient level of confidence to teach our program.

All of our results to date have been very encouraging. Students show a very high degree of motivation and learning. Students overwhelming think that the material is interesting and not too complex. In attitude assessments, we find a strong correlation between difficulty and interest: those parts of the course that are most difficult are also the most interesting. 


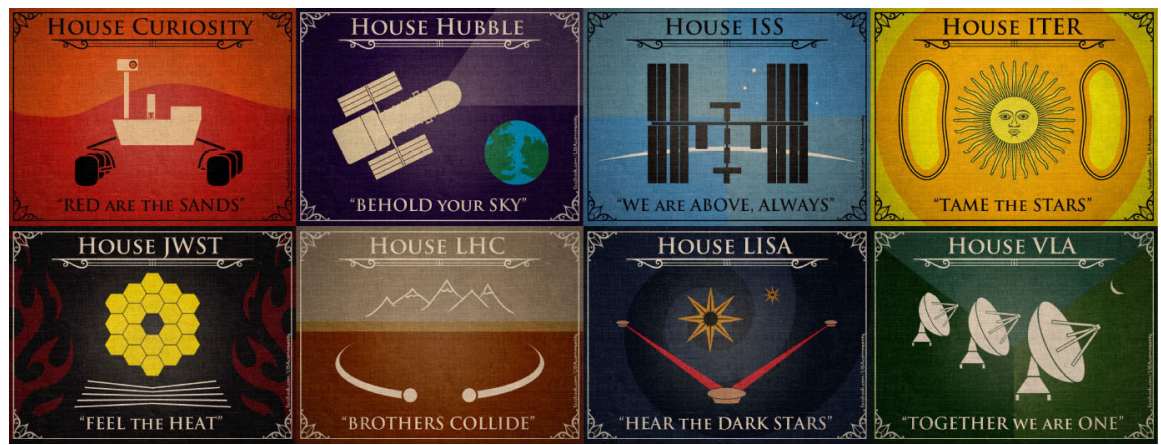

Fig. 1 "The houses of awesome exploration", an internet style meme that went viral and reached around two million people

\section{Social media outreach for eLISA (Presenter: O. Gerberding)}

At the beginning of 2013, a team of motivated scientists from the Albert Einstein Institute in Hannover started a social media outreach campaign to promote the proposed space-based gravitational-wave detector, eLISA. The aim of this activity was to increase awareness of the project and gravitational-wave astronomy in general. This was considered particularly important to counteract the negative attention generated by the financial problems of NASA in 2011, and the non-selection, in 2012, of the European-only version of LISA for the ESA L1 launch slot in 2022. The campaign also aimed to generate attention and support for the "Gravitational Universe" white paper [7] that was submitted on May the 24th 2013 in response to the L2/L3 mission call for science themes in the scope of the ESA Cosmic Vision Program.

The campaign was initialized by establishing a presence in four large social media platforms (Facebook, Google+, YouTube, Twitter). These were then provided with regular content updates to generate a community platform for experts and enthusiasts. The content included relevant scientific news, mission updates, and interactive games. Various meme-pictures were also generated and published. Such memes spread ideas through the internet, and are often imitated and altered during the process. One of our memes went viral trough a Facebook science blog and was seen by around two million people (see Fig. 1). The overall work load of the daily updates was roughly equivalent to one person full time.

Additionally, part of the team created three stop motion drawing videos under the brand Gravity Ink [8]. These videos, aimed at the interested layperson, explained parts of the science background for eLISA and gravitational wave astronomy. The style of the videos was based on the very successful minutephysics online video series (more than two million subscribers on YouTube) which also endorsed Gravity Ink. The three videos were viewed more than 100,000 times between May and November 2013. The production of each video required the equivalent of one full-time person for 1 week.

Non-scientific supporters were generated for the the Gravitational Universe white paper by promoting it on all platforms. The distributed computing project Einstein@Home [9] also promoted the white paper by featuring a dedicated news alert on 
the users' desktops. Based on their subscription comments we estimate that most of the supporters were gained through Einstein@Home and a maximum 5-10\% of them via the social media campaign. All together, 2800 non-scientific supporters registered on the dedicated webpage [10], together with around 1200 scientists.

\section{Teaching general relativity to undergraduates (Presenter: N. Christensen)}

Inspired by new results in cosmology and astrophysics, undergraduates are increasingly eager to learn about general relativity. Undergraduates are participating in gravitational wave research around the world, and their desire to learn and understand general relativity is coupled to both their research and education goals. Those who teach general relativity to undergraduates can take advantage of a decades worth of pedagogical progress. Various educational techniques long used in teaching electrodynamics and quantum mechanics to undergraduates can now be successfully applied to general relativity, and new textbooks are making such approaches broadly accessible. General relativity is therefore rapidly becoming as important as many topics traditionally covered for an undergraduate physics degree. We strongly recommend that any department offering a physics major consider a regular course offering in this fascinating subject. In our presentation we provided some ideas and advice in order to help make effective teaching of general relativity to undergraduates a reality. A comprehensive summary of results and conclusions pertaining to teaching general relativity to undergraduates can be found in [11].

Presently more and more universities and colleges are creating appropriate general relativity courses for undergraduate physics majors. These are typically single semester length courses. Many good textbooks are available. There are new strategies for introducing general relativity at a reasonable pace and level. These general relativity courses are not limited to the most gifted students. Undergraduates in 3rd or 4th year are quite capable of learning general relativity in satisfying depth. We suggest that all institutions offering an undergraduate physics degree consider providing a semester length general relativity course (it could be offered every other year, for example).

Historically general relativity was not taught to undergraduates since these students would not normally have studied differential geometry. However, we have found more and more approaches to teaching this subject to undergraduates. We identified four distinct approaches that the authors of textbooks have used to address present general relativity. We call them:

- Adjusted math first approach (see for example [12])

- Calculus only approach (see for example [13])

- Physics first approach (see for example [14])

- Intertwined + active learning approach (see for example [15])

We have identified a few tools for success in teaching general relativity to undergraduates. Prerequisites are important; for example, we recommend at least vector calculus, and a 2nd year modern physics course. Have students ask themselves what they can measure; make students think about taking measurements with meter sticks 
and clocks in a stationary or freely falling laboratory or in a distant observatory. Students need to understand the difference between global coordinates and real physical measurements performed in a local laboratory. Use active learning [16], and work as a team in solving general relativity problems. Finally, computer advances have made it possible for undergraduates to do calculations and explore physical problems that would have been daunting a decade ago. General relativity is a subject that undergraduates can successfully study!

\section{Inspiring undergraduate interest: aspects of NSF's IREU program (Presenter: B. Whiting)}

\subsection{Summary}

At a national level, the National Science Foundation (NSF) in the US is attempting to stem the tide of undergraduates turning irrevocably away from Physics before they have an opportunity to experience the excitement of research. Research Experiences for Undergraduates (REU) programs are designed to introduce students to research as early as possible so as to awaken, or sustain, an interest in a life dedicated to research. International REU (or IREU) programs such as ours (see http://www.phys. ufl.edu/ireu) go one step further, by exposing students to the international arena of research, awakening them to large-scale collaborative projects, and giving them an invaluable networking opportunity even before they enter graduate school. These programs entail dealing with many specific issues at both the organizational and host level. Understanding these issues, and developing creative ways to deal with them are crucial to the success of such programs. The maximum benefit for hosts also depends on a shrewd investment for success.

\subsection{IREU overview}

The international research experience for undergraduates (IREU) program at the University of Florida (UF) in gravitational-wave physics [17] is now in its eighth year. It is funded by US National Science Foundation (NSF), with Bernard Whiting as Director and PI and Guido Mueller as Co-PI. The focus of the program is on students from small schools with limited access to physics research resources, and students with minority status (which, in physics, especially includes women) seeking increased exposure to research. Our program is designed to expose US (and permanent resident) students to the rigors of gravitational physics research within a setting that truly reflects its international character. Students within related science communities are particularly welcome.

The NSF is anxious to increase the flux of young students, and minorities in particular, into a life of scientific research, a growth viewed as essential for future national economic prosperity. REU programs are designed to give undergraduates early exposure to what a life in research may be like, to allow them to experience the excitement it stimulates, and to encourage and support them to pursue it.

Each year we have a range of over fifty exciting projects [18] lined up at (currently nineteen) participating host sites around the world [19]. The research groups at 
these institutions are among the largest and most productive groups in the community. Affiliates of the LIGO or LISA science communities may also suggest other host institutions abroad, should they afford a better match to their research profile. For a period of 9-10 weeks during the summer, participants work on carefully selected research projects in some of the best gravitational physics labs in Europe and Australasia. During their stay, students also benefit from the valuable cultural enrichment that comes with living in a foreign environment.

Due to the long lead time required to make international arrangements for travel, accommodation and hosting in the many countries we use each Summer, applications open in mid-September and effectively close around December 15th. A shortlist of students is interviewed and the allocation process commences early in the new year. Throughout the Spring, participants contact their hosts to receive an assignment of reading material related to their selected project. At the end of May, a briefing meeting is held in which the students meet face-to-face, and present to each other their understanding of their assigned project. The months of June and July are spent working in research laboratories abroad. In mid-August, students attend a debriefing meeting in which they give their research presentation and submit a written report on their project and accomplishments. See all at [20].

Accepting the offer of a place for participating in our program is certainly a challenge for students. They will find themselves in a foreign country, often in a foreign language, in a very unfamiliar setting, generally with at most one other US participant simultaneously present, and very far from home and family. To help students become a cohesive group even before the program starts, we invite them all to join a select Facebook group, which seems to be of significant benefit. During the briefing meeting they are given the opportunity to explore an unfamiliar city together. Often, throughout their stay abroad, students plan further excursions together, such as sight-seeing in foreign cities or exploring the wilderness on week-ends and public holidays.

We have found that students are moved to apply to our program for a variety of reasons, but excitement at the program as outlined on our web-site is certainly the dominant reason they give. Many students come to our program by word of mouth from previous participants, while others find us through the NSF web-site or through other web searches. Invariably, students will not apply to our program unless it keeps coming to the top of their list as they assemble and plan their research options. Certainly, an interest in cutting edge science, and the broader field of astrophysics as it relates to gravitational wave research, are essential pre-requisites for a successful experience. As a bonus, students occasionally have the opportunity to attend a scientific meeting with others from their host institution.

\section{BiG waves: a different kind of gravitational-wave summer school (Presenter: I. Mandel)}

In the summer of 2012, the gravitational-wave group at the University of Birmingham, UK, organized a summer school with a rather unusual format. The Birmingham Gravitational-wave summer school (BiG Waves) [21] focused on active student involvement instead of the usual didactic approach. 
In lieu of lectures, students read and analyzed ten keys papers from the fields of gravitational-wave instrumentation, data analysis, and astrophysics. The papers, which started with the classic [22] and culminated with a search results paper [23], were provided to the students several months in advance of the school, to enable them to prepare for shotgun seminars. One of the main successes of the school was that students self-organized to study the papers together even before the start of the school. During the school, a student was randomly selected to lead the discussion of the key science topics of each paper at the board, with other students actively participating by filling in any gaps in the presentation, while the organizers acted as facilitators for the group discussion.

Students also engaged in two hands-on projects. On the experimental side, participants had the opportunity to build and program a stand-alone voice-recognition system using the Arduino chip. On the data analysis side, participants implemented an optimal matched filtering search and a Markov Chain Monte Carlo parameter estimation analysis on mock data. These projects were designed to provide students with practical experience in several data-analysis and experimental techniques (such as manipulation of large observational data sets, Bayesian inference, data acquisition, design of active control systems and digital electronics) that are likely to be useful across many areas of astrophysics. The students worked on these projects for the duration of the school. Each student took part in both projects, spending two to three hours per day on each project. The project results were presented and discussed during the final day of the school.

While beginning graduate students formed the primary target group for the school, participants ranged from undergraduates to senior graduate students and postdocs. More experienced participants often served as mentors to those with less experience in the relevant subject area, or contributed to designing the hands-on projects. Meanwhile, the breadth of the school ensured that everyone had an opportunity to learn, and the faculty members running the school moonlighted as beginning students for some of the projects.

We collected feedback on the school, both verbally and through entrance and exit questionnaires. Students gave the school an average ranking of 4.2 out of 5 for utility for their Ph.D. project, and reported that it motivated them to pursue an academic career. The feedback also highlighted a number of opportunities for further improvement. Challenges included keeping shotgun-seminar discussions from becoming lectures by the faculty, and ensuring that hands-on projects had a good connection with the reading activities and the right balance of exploration and implementation, while providing the satisfaction of yielding a completed, functional product.

After the GR-20/Amaldi conference, in September 2013, we repeated the BiG Waves school with modifications based on the feedback gained earlier. More details will be reported elsewhere [24].

\section{Conclusions}

The rich variety of activities highlighted in this parallel session indicates that a vibrant and enthusiastic education and public outreach community now exists within this field. 
While outreach in the area of "Einsteinian physics" can be challenging, many of these activities are proving very successful and are helping to challenge the conventional paradigm that the teaching of concepts such as general relativity and gravitational waves to non-specialists is too difficult. In addition the growing body of new and innovative internet-based outreach is beginning to engage the public in very large numbers. As we prepare to open fully this new window on the Universe, it seems clear that education and public outreach on gravitational-wave astronomy is in very good health.

Acknowledgments The session convenors Martin Hendry and Carlo Bradaschia would like to thank all of the speakers and everyone who participated in the session for making it a success. The work of NC was supported by NSF grant PHY-1204371. This program is currently supported by grant PHY-1005036 from the NSF.

\section{References}

1. Stuver, A.: Living LIGO blog http://livingligo.org

2. Discovery blog: Gravitational Affairs: LIGO's Little Black Box. http://news.discovery.com/space/ ligos-little-black-box-110317.htm

3. Andrews, B.: 20 Things You Didn’t Know About Gravity. Discover Magazine, July/August 2013. http://tinyurl.com/discovergravity (2013)

4. Stuver, A.: A LaserFest Teachers' Day. APS Forum on Education Spring Newsletter. http://www.aps. org/units/fed/newsletters/spring2011/stuver.cfm (2011)

5. Cavaglia, M., Hendry, M.A., Ingram, D., Milde, S., Reitze, D., Riles, K., Schutz, B., Stuver, A.L., Summerscales, T., Thacker, J., Torres, C.V., Ugolini, D., Vallisneri, M., Zermeno, A.: Gravitationalwave astronomy: opening a new window on the universe for students, educators and the public. ASP Conf. Ser. 400, 328 (2008)

6. Pitts, M., Venville, G., Blair, D.G., Zadnik, M.: Teaching aspects of Einsteins general theory of relativity in year 6: an exploratory case study. Res. Sci. Educ. (2014). doi:10.1007/s11165-013-9386-6

7. Danzmann, K., et al.: The Gravitational Universe. arxiv.org/abs/1305.5720 (2013)

8. Gravity Ink. video series. http://tinyurl.com/gravityink

9. Einstein@Home. einsteinathome.org

10. The Gravitational Universe. https://support.elisascience.org

11. Christensen, N., Moore, T.: Teaching general relativity to undergraduates. Phys. Today 65(6), 41 (2012)

12. Taylor, E.F., Wheeler, J.A.: Exploring Black Holes: Introduction to General Relativity. Addison Wesley Longman, San Francisco (2000)

13. Schutz, B.: A First Course in General Relativity, 2nd edn. Cambridge University Press, New York (2009)

14. Hartle, J.B.: Gravity: An Introduction to Einstein's General Relativity. Addison-Wesley, Reading (2003)

15. Moore, T.: A General Relativity Workbook. University Science Books, Sausalito (2012)

16. Hake, R.R.: Interactive-engagement versus traditional methods: a six-thousand-student survey of mechanics test data for introductory physics courses. Am. J. Phys. 66, 64 (1998)

17. Gravitational Physics IREU website. http://www.phys.ufl.edu/ireu

18. Gravitational Physics IREU projects. http://www.phys.ufl.edu/ireu/projects

19. Gravitational Physics IREU host institutions. http://www.phys.ufl.edu/ireu/hosts

20. Gravitational Physics IREU reports. http://www.phys.ufl.edu/ireu/reports

21. Birmingham Gravitational Waves Summer School. http://www.sr.bham.ac.uk/dokuwiki/doku.php? id=bigwaves:bigwaves 2012

22. Schutz, B.F.: Am. J. Phys. 42, 412 (1984)

23. Abadie, J., et al. (LIGO Scientific Collaboration): Phys. Rev. D 83, 122005 (2011)

24. Mandel, I., Farr, W., Freise, A., Vecchio, A.: (in preparation) 